\title{
Targeting of Peptidergic Vesicles in Cotransmitting Terminals
}

\author{
Tuula Karhunen, Ferdinand S. Vilim, Vera Alexeeva, Klaudiusz R. Weiss, and Paul J. Church \\ Department of Physiology and Biophysics, Mount Sinai School of Medicine, New York, New York 10029
}

In the present study, we examined the targeting of neuropeptide-containing vesicles in terminals of neurons that release both neuropeptides and classical transmitters. Single neurons were electrically stimulated with patterns of activity that were recorded in freely behaving animals. The amount of peptide release was measured biochemically using a radioimmunoassay, and the targeting of peptidergic vesicles was quantified using immunoelectronmicroscopy. Repeated electrical stimulation of single neurons produced a very large increase in peptide release. Peptide release is paralleled by a twofold increase in the number of peptidergic vesicles docked at the portion of the terminal membrane that is away from the target muscle. This is in stark contrast to cholinergic vesicles, which aggregate at, and are released from the conventional release sites in close apposition to the muscle. This differential targeting of cholinergic and peptidergic vesicles may play a significant role in the distinct release requirements and spatial and temporal characteristics of the actions of conventional and peptidergic transmitters.

Key words: cotransmission; synaptic transmission; peptide; presynaptic terminal; facilitation; DCV; LDCV
Many neurons contain both classical and peptidergic transmitters that are thought to be released under different conditions and to transmit distinct types of information (Hokfelt, 1991; Kupfermann, 1991). Classical transmitters, concentrated in small clear vesicles (SCVs), act in a rapid and spatially restricted manner. This necessitates that SCVs be specifically targeted to the active zones that lie in close apposition to transmitter targets. In contrast, neuropeptides exert slower actions and act over much larger time scales and distances (Jan and Jan, 1982; Zupanc, 1996). These characteristics of peptide actions do not appear to necessitate the targeting or release of peptide-containing large dense core vesicles (LDCVs) from the active zones.

Although the site of release may significantly affect both the requirements for release and the characteristics of transmitter actions, it has been difficult to determine whether, under physiological conditions, LDCVs are specifically targeted to distinct parts of the cotransmitting presynaptic terminal. Some previous studies failed to demonstrate that the LDCVs actually were peptidergic (Dickinson-Nelson and Reese, 1983; Pow and Golding, 1987; Pecot-Dechavassine and Brouard, 1997). Others, because of the low abundance of LDCVs, relied on nonphysiological stimulation methods (e.g., high $\mathrm{K}^{+}$) that maximize release (Buma and Roubos, 1986; Morris and Pow, 1991). Results from many studies in a variety of preparations suggest that all parts of the neuronal membrane are competent to release LDCVs (Morris and Pow, 1991). However, this competence does not mean that, under physiological conditions, the LDCVs are nonselectively targeted and/or released. Nonphysiological stimulation could elevate calcium in parts of a neuron in which calcium elevation does not occur during normal spike activity, thus lead-

\footnotetext{
Received Sept. 11, 2000; revised Nov. 20, 2000; accepted Nov. 21, 2000.

This research was supported by National Institute of Health Grants K01 MH01624 (P.J.C.), DA13330 (F.S.V.), MH36730, and K05 MH01427 (K.R.W.). We thank Dr. E. C. Cropper, Dr. V. Brezina, and N. Dembrow for their critical reading of an earlier version of this manuscript.

Correspondence should be addressed to Dr. Paul J. Church, Department of Physiology and Biophysics, Box 1218, Mount Sinai School of Medicine, 1 Gustave Levy Place, New York, NY 10029. E-mail: pchurch@inka.mssm.edu.

Copyright (C) 2001 Society for Neuroscience $0270-6474 / 01 / 210001-\bullet \$ 15.00 / 0$
}

ing to artifactual targeting and release of peptides. Importantly, the apparently generalized competence of the membrane for peptide release suggests that if LDCVs are to be released from specific areas of neuronal membranes, the preferential targeting of LDCVs to those areas may constitute the critical step for selectivity. In this study we demonstrate such preferential targeting under conditions of normal neuronal activity.

\section{MATERIALS AND METHODS}

Aplysia californica $(\sim 225 \mathrm{gm})$ were maintained at $14-16^{\circ} \mathrm{C}$ on a $12 \mathrm{hr}$ light/dark cycle and food-deprived $\geq 3 \mathrm{~d}$. Before dissection animals were injected with isotonic $\mathrm{MgCl}_{2}(25-50 \%$ body wt). The semi-intact neuromuscular preparation including the buccal ganglion and the accessory radular closer muscle (ARC) was isolated as described elsewhere (Vilim et al., 1996). Briefly, the buccal ganglion was pinned in a dish containing $25 \%$ isotonic $\mathrm{MgCl}_{2}$ to prevent spontaneous activity, and buccal nerve 2 was passed through a slit in the side of the dish. The ARC was suspended outside the dish and perfused directly through an artery. Before the beginning of each experiment, the preparation was perfused with artificial seawater for a minimum of $2 \mathrm{hr}$. Therefore, it is likely that any soluble protease found in the hemolymph would be washed out of the preparation (Whim and Lloyd, 1989). Individual drops of perfusate were collected every $2.5 \mathrm{~min}$ into the tubes in which the radioimmunoassay (RIA) was performed so that no losses because of peptide handling occurred. Identified B15 motor neurons were impaled with two microelectrodes, one to inject current, and one to monitor membrane potential. The temperature was maintained at $15 \pm 0.5^{\circ} \mathrm{C}$ throughout the experiment.

This article is published in The Journal of Neuroscience, Rapid Communications Section, which publishes brief, peer-reviewed papers online, not in print. Rapid Communications are posted online approximately one month earlier than they would appear if printed. They are listed in the Table of Contents of the next open issue of JNeurosci. Cite this article as: JNeurosci, 2001, 21:RC127 (1-5). The publication date is the date of posting online at www.jneurosci.org.

http://www.jneurosci.org/cgi/content/full/4960 
Radioimmunoassays. A standard RIA (Vilim et al., 1996) was used to quantify the release of peptides. The rabbit antibody to $\mathrm{SCPb}$ used for RIA was a gift from Dr. H. R. Morris (Imperial College, London, UK). $\mathrm{SCPb}$ was synthesized by AnaSpec (San Jose, CA). Desaminotyrosinated $\mathrm{SCPb}$ was iodinated $\left({ }^{125} \mathrm{I}\right)$ using the chloramine T method. The amount of peptide collected in each sample was determined by comparison of counts bound in the experimentals to those generated by a standard curve. Because LDCVs in B15 contain both the SCPs (including SCPa and $\mathrm{SCPb}$ ), and the buccalins (BUCs) and both peptide families are coreleased (Vilim et al., 1996), detection of SCPb release indicates the release of $\mathrm{SCPa}$ and the BUCs as well. Because of the between animal variability in total peptide release, the data were normalized to the total release in each experiment.

EM immunocytochemistry. The ARC muscle was fixed during stimulation by perfusing the muscle via the artery with $4 \%$ glutaraldehyde. The tissue was then processed as previously described (Vilim et al., 1996). Briefly, tissue was fixed (4\% glutaraldehyde, $10 \%$ sucrose, $11 \mathrm{~mm}$ magnesium chloride, and $0.2 \mathrm{M} \mathrm{Na}$ HEPES, pH 7.6) for $3 \mathrm{hr}$ (RT), post-fixed with $1 \%$ osmium tetroxide in buffer at $4^{\circ} \mathrm{C}$ for $1 \mathrm{hr}$, and embedded in EMbed 812 (Electron Microscopy Sciences, Fort Washington, PA). Tissue sections were immunostained with a primary antibody to $\mathrm{SCPb}$ (Berkeley Antibody Company, Richmond, CA) and gold-labeled secondary antibodies, to unequivocally identify B15 terminals. Ultrathin sections were examined and photographed with a Zeiss $\mathrm{CH}-10$ electron microscope at $60 \mathrm{kV}$. Electron microscopy supplies and reagents were obtained from Electron Microscopy Sciences.

\section{RESULTS}

The question of whether LDCVs are targeted differently than the SCVs can be best answered by studying cotransmitting neurons. We used the Aplysia motor neuron B15, which releases both ACh and neuropeptides (Cohen et al., 1978; Lloyd et al., 1987; Cropper et al., 1990a; Vilim et al., 1996). This preparation offers several advantages, e.g., peptide release can be measured under physiological conditions of B15 stimulation, B15 terminals can be unequivocally identified, and the LDCVs in these terminals have been shown to contain the neuropeptide SCP (Vilim et al., 1996). These features make it possible to relate peptide release to LDCV distribution.

\section{Dynamics of peptide release}

B15 was stimulated with patterns of activity that were previously recorded in freely behaving animals (Cropper et al., 1990b). Low levels of peptide release were detected only after several minutes of stimulation, but with continued stimulation, peptide release facilitated dramatically ( $\sim 100$ times) peaking at $\sim 40 \mathrm{~min}$ and then slowly declining over the next 20 min (Fig. 1). Several lines of evidence indicate that the delay, facilitation, and defacilitation of recovered peptide is an accurate reflection of the dynamics of peptide release, and not an artifact caused by protease activity or a time lag in the clearance of peptides from the muscle in this preparation. Previously published results demonstrated that the SCPs and Buccalins are colocalized to LDCVs in B15 terminals and exhibit very similar release kinetics (Vilim et al., 1996). In addition, although the absolute amount of SCP released by B15 varies dramatically from preparation to preparation, the kinetics of release are very consistent (Vilim et al., 1996). Finally, in facilitated preparations, peptide release is easily detected within the first $2.5 \mathrm{~min}$ of stimulation (Vilim et al., 1996). Thus, once released from the terminals, there is no significant barrier to the efflux of peptides into the perfusate. The absence of a diffusion barrier is perhaps not surprising because the circulatory system of the animal is half open and does not involve a venous return.

Stimulation was terminated at $60 \mathrm{~min}$, coincident with the collection of a drop of perfusate. Therefore, the next drop, which occurs at $62.5 \mathrm{~min}$., contains peptide released during the stimulation period. From there the amount of peptide in the perfusate

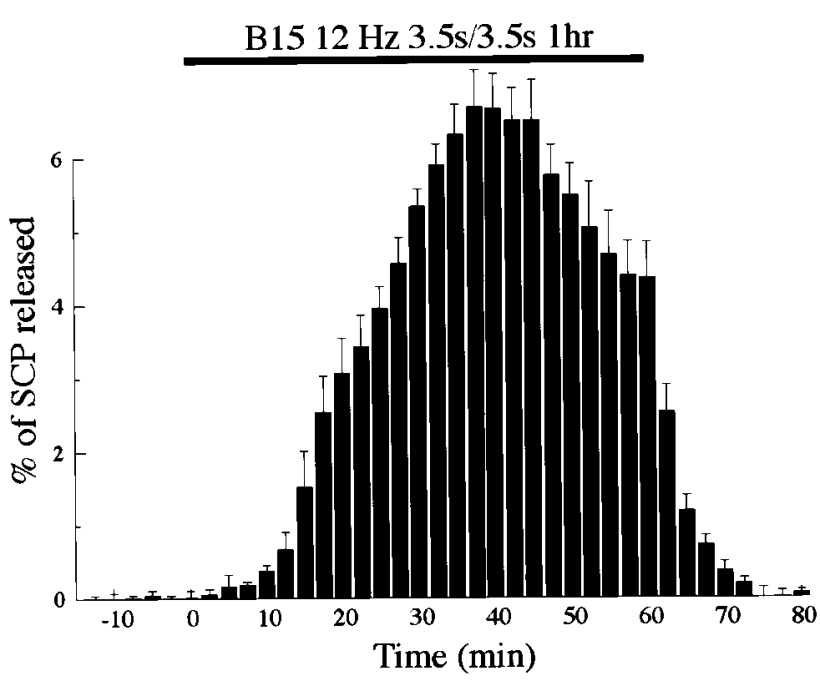

Figure 1. Release of SCP in response to stimulation of motor neuron B15. During the period indicated by the bar, B15 was fired at $12 \mathrm{~Hz}$ for 3.5 sec every $7 \mathrm{sec}$. Samples of ARC perfusate were collected every $2.5 \mathrm{~min}$ and analyzed by RIA for their peptide content. SCP release is expressed as percentage of total release in each experiment (mean \pm SEM; $n=5$ ). Peptide release was detected by $10 \mathrm{~min}$ and then facilitated greatly, peaked at $\sim 40 \mathrm{~min}$, and slowly declined until stimulation was terminated at $60 \mathrm{~min}$.

drops precipitously, and by $10 \mathrm{~min}$ is reduced to $\sim 5 \%$ of maximum release. Although a determination is beyond the temporal resolution of our assay, a portion of the peptide recovered after stimulation has ceased could represent spontaneous or "delayed release" of peptide. Indeed, after patterns of stimulation that lead to increased transmitter release (e.g., PTP) the evoked or "phasic" release of conventional transmitters such as $\mathrm{ACh}$ is often followed by an increase in spontaneous or "delayed" release (Barrett and Stevens, 1972; Rahamimoff and Yaari, 1973; Zucker and Lara-Estrella, 1983; Cohen and Van der Kloot, 1986; Goda and Stevens, 1994; Atluri and Regehr, 1998).

\section{Targeting of LDCVs}

To characterize LDCV targeting we compared their distribution in micrographs from unstimulated (Fig. $2 A$ ) and $30 \mathrm{~min}$ stimulated (Fig. $2 B$ ) preparations. The 30 min time point was chosen to maximize the likelihood of significant LDCV redistribution while minimizing contaminating effects of possible LDCV depletion. Presynaptic terminals were identified by enriched content of LDCVs and SCVs and highly parallel regions of apposition between the neuronal membrane and the muscle membrane. These membrane regions appeared more electron-dense and were associated with an accumulation of SCVs near the presynaptic membrane, indicative of the active zones or conventional release sites. After extended stimulation, we observed an apparent depletion of the SCVs, and therefore the enriched LDCV content and the electron-dense parallel membranes became the predominant criteria for identifying the terminal regions. Presynaptic terminals were unequivocally identified as those of B15 by immunostaining with SCP antibodies. Previous work has shown that in the ARC neuromuscular system only LDCVs contained in B15 stain for SCP (Vilim et al., 1996).

From each preparation the first six terminals that contained at least seven LDCVs were analyzed. For the purpose of our analysis we divided the membrane of the terminal region into two parts, the membrane that is in apposition to the muscle [muscle face $(\mathrm{MF})$ ], which includes the conventional release sites, and the 

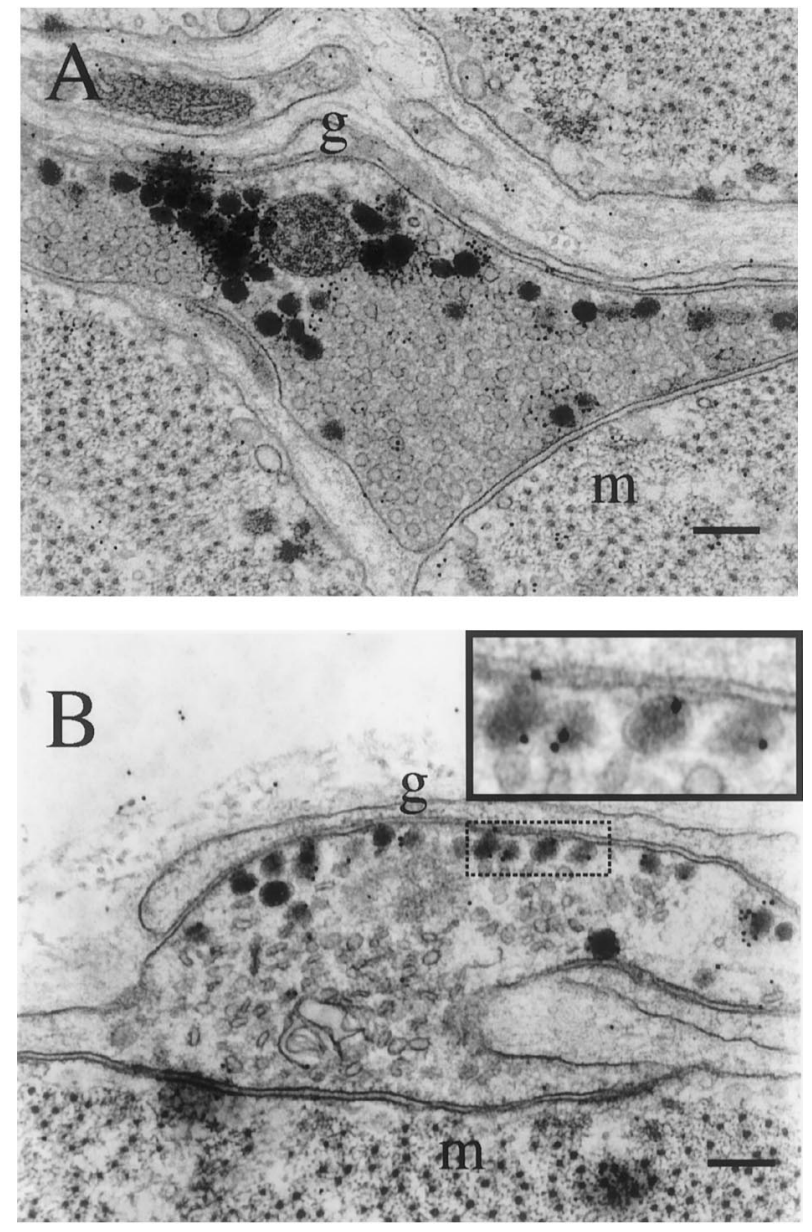

Figure 2. Morphological correlate to peptide release. Photomicrographs from unstimulated $(A)$ and stimulated $(B)$ preparations. SCP immunoreactivity $(B$, inset) was used to unequivocally identify the terminals as those of B15 terminals, to identify individual LDCVs as SCP-containing, and to insure that the LDCVs analyzed are not endocytotic. In unstimulated preparations, LDCVs are almost entirely excluded from the MF of the terminal that is packed with SCVs. After $30 \mathrm{~min}$ of stimulation there is an increase in the number of LDCVs within $55 \mathrm{~nm}$ of the NMF and still very few LDCVs near the MF. After $30 \mathrm{~min}$ of stimulation we observed an increase in the number of small (@40 $\mu \mathrm{m}$ diameter), and occasionally large $(\cong 100 \mu \mathrm{m}$ diameter $)$ irregularly shaped, clear membrane-bound vacuoles dispersed throughout the cytoplasm $(B)$. Although we have no direct evidence, these may be the result of endocytosis. $g$, Glia; $m$, muscle. Scale bar, $200 \mathrm{~nm}$.

membrane that does not appose muscle [nonmuscle face (NMF)]. This avoids possible ambiguities concerning the exact limits of the active zone. We then measured the distance from each LDCV to the nearest membrane, MF or NMF. Because the largest cross sections of LDCVs observed in this study were $110 \mathrm{~nm}$ in diameter, a distance of $55 \mathrm{~nm}$ from the center point of the LDCV to the membrane could be defined as morphologically docked. Data were grouped into $55 \mathrm{~nm}$ bins for analysis. Because of the size and shape of the terminals, beyond a distance of $220 \mathrm{~nm}$ some of the LDCVs were actually closer to the opposite membrane. Therefore, to avoid double counting of LDCVs, data for those located $\leq 220 \mathrm{~nm}$ from either membrane are shown.

In unstimulated preparations, $>70 \%$ of the LDCVs are located within $220 \mathrm{~nm}$ of the NMF (Fig. 3A1). Furthermore, the distribution LDCVs within this range exhibits a striking asymmetry $\left(\chi^{2}=11.6 ; p<0.01\right)$. Whereas relatively few LDCVs are morphologically docked $(8 \pm 3)$, a large number of LDCVs are
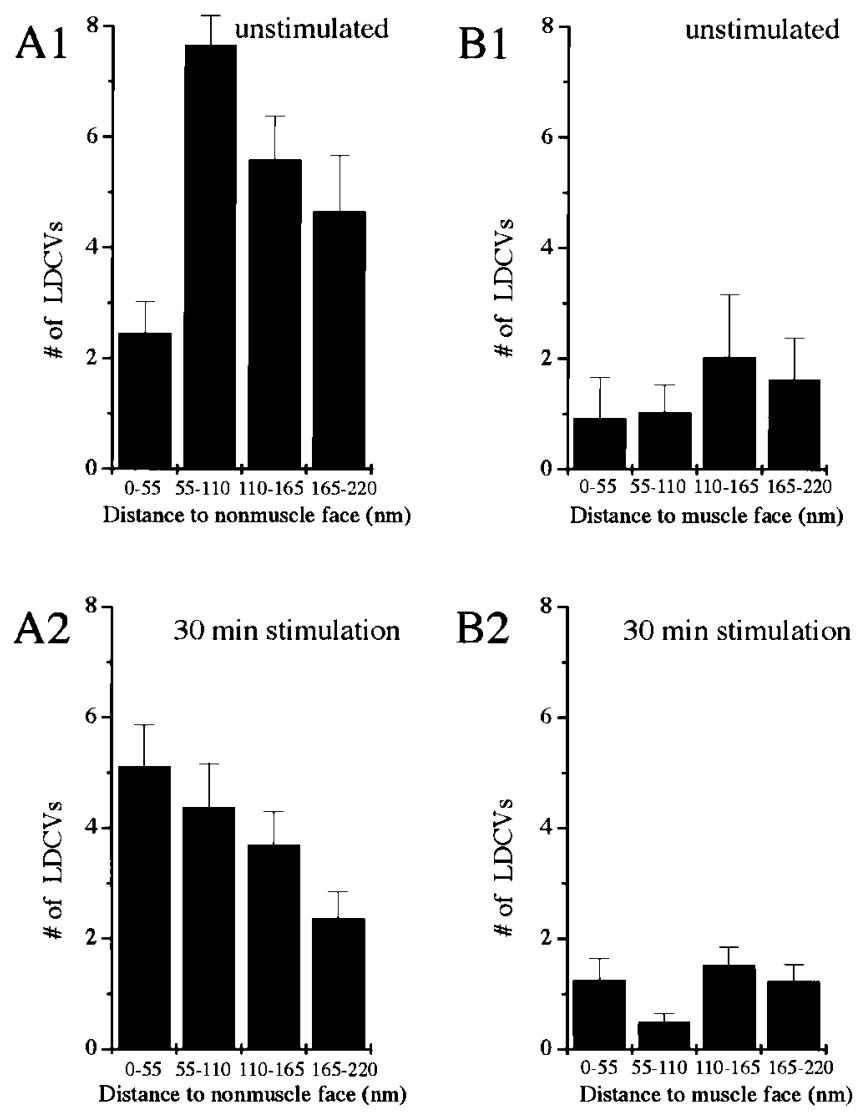

Figure 3. Distribution pattern of LDCVs within the presynaptic terminal. The shortest distance of each LDCV to the MF and NMF membranes was measured (center point of LDCV to membrane). For each experiment, the data were grouped into four $55 \mathrm{~nm}$ bins and expressed as absolute number of LDCVs per bin (for each, mean \pm SEM; $n=5$ ). $A$, Distribution of LDCVs within $220 \mathrm{~nm}$ of NMF in unstimulated $(A 1)$ and stimulated $(A 2)$ preparations. B, Distribution of LDCVs within $220 \mathrm{~nm}$ of $\mathrm{MF}$ in unstimulated $(B 1)$ and stimulated $(B 2)$ preparations. In unstimulated preparations, relatively few LDCVs are morphologically docked (within $55 \mathrm{~nm}$ of the terminal membrane), however a large number of LDCVs are located 55-110 nm from the NMF $(A 1)$. In contrast, relatively few $(<25 \%)$ of the LDCVs are located in the vicinity of the MF $(B 1)$, and those LDCVs are evenly distributed $\left(\chi^{2}=0.57 ; p>0.9\right)$. After $30 \mathrm{~min}$ of stimulation there is a significant increase in the number of LDCVs docked at the NMF $(B 1)(p<0.01)$, although the total number of LDCVs is decreased. In contrast, there is no apparent redistribution of LDCVs in the vicinity of MF $(B 2)$ ( $p>0.3$ for each of the bins).

located 55-110 nm from the NMF, and the proportion declines over the next two bins (Fig. 3A1). In contrast, relatively few $(<25 \%)$ of the LDCVs are located in the vicinity (within $220 \mathrm{~nm}$ ) of the MF, and those vesicles are evenly distributed across that range $\left(\chi^{2}=0.57 ; p>0.9\right)$. It is worth noting, that for each terminal analyzed, with one exception, the length of the NMF exceeded that of the MF (the ratio was 2.3:1 \pm 0.02 ). Even when the LDCV distribution was normalized to the length of membranes the LDCVs were more than twice as abundant at the NMF than the MF, indicating that before stimulation, the LDCVs are preferentially located near the NMF.

After 30 min of stimulation, there is an $\sim 25 \%$ reduction in the total number of LDCVs in the B15 terminals (control, $31 \pm 2.9$; 30 min stimulation, $23.6 \pm 2.0$; LDCVs per terminal, mean \pm SEM; for each, $n=30$ ). This is not surprising, given the large amount of peptide release. More interesting however, is the dramatic redistribution of LDCVs near the NMF. Specifically, even in the face of a reduction in the total number of LDCVs in 
the terminal, there is a significant increase in the absolute number of docked LDCVs at the NMF $(p<0.01)$ (Fig. 3A2). In addition, there is an associated decrease in the number of LDCVs within the 55-220 nm range $(p<0.05)$. In other words, $>27 \%$ of the remaining LDCVs are now docked at the NMF (opposed to $\sim 8 \%$ docked at the NMF in unstimulated preparations). In contrast, there is no significant redistribution of LDCVs near the $\mathrm{MF}$ and no increase in the number of LDCVs docked at the MF ( $p>0.3$ for each of the bins) (Fig. 3B2). The differences in the redistribution of LDCVs cannot be attributed to stimulationevoked changes in the ratio of the NMF: MF membrane length as this ratio was unchanged $(p>0.5)$.

\section{DISCUSSION \\ Dynamics of peptide release}

During continued stimulation, release of SCP from B15 is not constant, but rather exhibits a great degree of plasticity. Whereas the characterization of the mechanisms underlying the dynamics of peptide release is beyond the scope of this study, a few points are worth noting. The observed delay to onset of peptide recovery is not an artifact of the assay, because there is no such delay in previously facilitated preparations (Vilim et al., 1996). The relatively low number of LDCVs "docked" in previously unstimulated preparations may contribute to the delay in onset of peptide release (see below). The large facilitation may be attributable to a number of mechanisms, including changes in ionic conductances leading to spike broadening (and thus an increase in $\mathrm{Ca}^{2+}$ influx), and changes in the efficacy or $\mathrm{Ca}^{2+}$ sensitivity of the protein "machinery" involved in peptidergic transmission, as has been demonstrated for the facilitation of conventional transmitter release (Byrne and Kandel, 1996). The observed defacilitation after $40 \mathrm{~min}$ of stimulation may be the result of the actions of inhibitory peptides released by this neuron (Vilim et al., 1996), the possible depletion of a releasable pool of LDCVs, or even failure of action potential propagation (Branchaw et al., 1998). This last possibility is less likely in this system, however, because muscle contractions, which are extremely sensitive to changes in spike frequency and number, were constantly monitored and exhibited no signs of spike failure.

\section{LDCV distribution in nerve terminals}

In the absence of stimulation, LDCVs are not randomly distributed throughout the B15 presynaptic terminal, but rather they are almost entirely excluded from the active zones, which are packed with SCVs. In addition, unlike SCVs, many of which are morphologically docked at active zones, very few LDCVs are docked at either the MF or the NMF. Indeed, a large number of LDCVs are located between 55 and $110 \mathrm{~nm}$ of the terminal membrane away from the muscle. The mechanism underlying this segregation of SCVs and DCVs is unclear. Although we have no direct evidence, the relatively low number of docked LDCVs is consistent with the presence of a cystoskeletal barrier, as has been proposed in endocrine cells (for review, see Trifaro et al., 2000). Such a barrier could also contribute to the delayed onset of peptide release. The exclusion of LDCVs from the active zones could simply be a consequence of the specific targeting of SCVs to the active zones. The large number and density of SCVs at the active zone could be sufficient to exclude LDCVs from that area of the terminal. However, very few LDCVs are located in the vicinity of the active zones even after the apparent depletion of SCVs that follows prolonged stimulation (see below). It is possible that the large number of LDCVs localized to a region within the presynaptic terminal between 55 and $110 \mathrm{~nm}$ of the NMF is the result of specific tethering of the LDCVs to cytosolic elements, such as the actin cytoskeleton. Indeed, studies in other systems suggest that rather than being located near the conventional release sites, LDCVs are located near the $\mathrm{Ca}^{2+}$ release sites of internal stores (Lysakowski et al., 1999; Tse and Tse, 1999).

Whether the result of simple exclusion from the active zone, a cystoskeletal barrier, or specific targeting to cytosolic elements of the presynaptic terminal, the differential distribution of LDCVs from SCVs within the presynaptic terminal has clear implications regarding the different release requirements for conventional and peptide transmitters. Although conventional transmitter release can be evoked by a single action potential, it requires a relatively high elevation of intracellular calcium (tens of micromolar) which only occurs in microdomains at the active zones near the site of calcium entry (Zucker, 1996). Conversely, whereas a 10-fold lower calcium elevation is required for peptide release, it is generally believed that a burst of action potentials is required to evoke peptide release (Zupanc, 1996; however, see Whim et al., 1997). Indeed, previous studies have shown that motor neuron $\mathrm{B} 15$ can release its conventional transmitter ( $\mathrm{ACh}$ ) in response to a single action potential, but a burst of action potentials is required to evoke peptide release (Cohen et al., 1978; Whim and Lloyd, 1989; Vilim et al., 1996). In the present study, the differential distribution of SCVs and LDCVs within the presynaptic terminal before stimulation may contribute to these different stimulation requirements for release.

\section{Redistribution of LDCVs in stimulated nerve terminals}

Because transmitter release requires that vesicles be brought to the membrane, one may expect to observe an increased number of docked LDCVs during conditions of facilitated release. Facilitated release was indeed associated with an increased number of docked LDCVs, but this increase was restricted to the NMF. This result strongly suggests that although SCP is known to act directly on the muscle, it is preferentially released away from muscle. This is perhaps not surprising given that previous studies have shown that peptide receptors can be located at some distance from the site of peptide release (Liu et al., 1994; Zupanc, 1996).

It has been proposed that terminals that contain multiple transmitters can work as closed, or "wired", synapses for one transmitter and as open, or "volume", synapses for another (Zoli and Agnati, 1996; Zoli et al., 1999). The specific targeting of LDCVs in B15 terminals away from conventional release sites may contribute to its ability to act in these dual synaptic roles. Specifically, whereas the fast-acting conventional transmitter $\mathrm{ACh}$ is released from active zones in close apposition to the target muscle, the release of SCP outside of the restricted space of the synaptic cleft may facilitate its diff usion and thus contribute to its ability to act over a greater target area. Finally, the ability to release combinations of transmitters under varying conditions confers a degree of synaptic plasticity unattainable with a single transmitter (Brezina and Weiss, 1997). The fact that distinct classes of transmitters exert their actions over different distances and time scales, are released under different conditions, and from different areas of the presynaptic terminal further enhances the range of plasticity.

\section{REFERENCES}

Atluri PP, Regehr WG (1998) Delayed release of neurotransmitter from cerebellar granule cells. J Neurosci 18:8214-8227. 
Barrett EF, Stevens CF (1972) The kinetics of transmitter release at the frog neuromuscular junction. J Physiol (Lond) 227:691-708.

Branchaw JL, Hsu SF, Jackson MB (1998) Membrane excitability and secretion from peptidergic nerve terminals. Cell Mol Neurobiol 18:45-63.

Brezina V, Weiss KR (1997) Analyzing the functional consequences of transmitter complexity. Trends Neurosci 20:538-543.

Buma P, Roubos EW (1986) Ultrastructural demonstration of nonsynaptic release sites in the central nervous system of the snail Lymnaea stagnalis, the insect Periplaneta americana, and the rat. Neuroscience 17:867-879.

Byrne JH, Kandel ER (1996) Presynaptic facilitation revisited: state and time dependence. J Neurosci 16:425-435.

Cohen IS, Van der Kloot W (1986) Facilitation and delayed release at single frog neuromuscular junctions. J Neurosci 6:2366-2370.

Cohen JL, Weiss KR, Kupfermann I (1978) Motor control of buccal muscles in Aplysia. J Neurophysiol 41:157-180.

Cropper EC, Miller MW, Vilim FS, Tenenbaum R, Kupfermann I, Weiss KR (1990a) Buccalin is present in the cholinergic motor neuron B16 of Aplysia and it depresses accessory radula closer muscle contractions evoked by stimulation of B16. Brain Res 512:175-179.

Cropper EC, Price DA, Tenenbaum R, Kupfermann I, Weiss KR (1990b) Release of peptide cotransmitters from a cholinergic motor neuron under physiological conditions. Proc Natl Acad Sci USA 87:933-937.

Dickinson-Nelson A, Reese TS (1983) Structural changes during transmitter release at synapses in the frog sympathetic ganglion. J Neurosci 3:42-52.

Goda Y, Stevens CF (1994) Two components of transmitter release at a central synapse. Proc Natl Acad Sci USA 91:12942-12946.

Hökfelt T (1991) Neuropeptides in perspective: the last ten years. Neuron 7:867-879.

Jan LY, Jan YN (1982) Peptidergic transmission in sympathetic ganglia of the frog. J Physiol (Lond) 327:219-246.

Kupfermann I (1991) Functional studies of cotransmission. Physiol Rev 71:683-732.

Liu H, Brown JL, Jasmin L, Maggio JE, Vigna SR, Mantyh PW, Basbaum AI (1994) Synaptic relationship between substance P and the substance $\mathrm{P}$ receptor: light and electron microscopic characterization of the mismatch between neuropeptides and their receptors. Proc Natl Acad Sci USA 91:1009-1013.

Lloyd PE, Frankfurt M, Stevens P, Kupfermann I, Weiss KR (1987) Biochemical and immunocytological localization of the neuropeptides FMRFamide, SCPA, SCPB, to neurons involved in the regulation of feeding in Aplysia. J Neurosci 7:1123-1132.
Lysakowski A, Figueras H, Price SD, Peng YY (1999) Dense-cored vesicles, smooth endoplasmic reticulum, and mitochondria are closely associated with nonspecialized parts of plasma membrane of nerve terminals: implications for exocytosis and calcium buffering by intraterminal organelles. J Comp Neurol 18:378-390.

Morris JF, Pow DV (1991) Widespread release of peptides in the central nervous system: quantitation of tannic acid-captured exocytoses. Anat Rec 231:437-445.

Pecot-Dechavassine M, Brouard M-O (1997) Large dense-core vesicles at the frog neuromuscular junction: characteristics and exocytosis. J Neurocytol 26:455-465.

Pow DV, Golding DW (1987) "Neurosecretion" by aminergic synaptic terminals in vivo: a study of secretory granule exocytosis in the corpus cardiacum of the flying locust. Neuroscience 22:1145-1149.

Rahamimoff R, Yaari Y (1973) Delayed release of transmitter at the frog neuromuscular junction. J Physiol (Lond) 228:241-257.

Trifaro J, Rose SD, Lejen T, Elzagallaai A (2000) Two pathways control chromaffin cell cortical F-actin dynamics during exocytosis. Biochimie 82:339-352.

Tse FW, Tse A (1999) Regulation of exocytosis via release of $\mathrm{Ca}^{2+}$ from intracellular stores. Bioessays 21:861-865.

Vilim FS, Price DA, Lesser W, Kupfermann I, Weiss KR (1996) Costorage and corelease of modulatory peptide cotransmitters with partially antagonistic actions on the accessory radula closer muscle of Aplysia californica. J Neurosci 16:8092-8104.

Whim MD, Lloyd PE (1989) Frequency-dependent release of peptide cotransmitters from identified cholinergic motor neurons in Aplysia. Proc Natl Acad Sci USA 86:9034-9038.

Whim MD, Niemann H, Kaczmarek LK (1997) The secretion of classical and peptide cotransmitters from a single presynaptic neuron involves a synaptobrevin-like molecule. J Neurosci 17:2338-2347.

Zoli M, Agnati LF (1996) Wiring and volume transmission in the central nervous system: the concept of closed and open synapses. Prog Neurobiol 49:363-380.

Zoli M, Jansson A, Sykova E, Agnati LF, Fuxe K (1999) Volume transmission in the CNS and its relevance for neuropsychopharmacology. Trends Pharmacol Sci 20:142-150.

Zucker RS (1996) Exocytosis: a molecular and physiological perspective. Neuron 17:1049-1055.

Zucker RS, Lara-Estrella LO (1983) Post-tetanic decay of evoked and spontaneous transmitter release and a residual-calcium model of synaptic facilitation at crayfish neuromuscular junctions. J Gen Physiol 81:355-372.

Zupanc GK (1996) Peptidergic transmission: from morphological correlates to functional implications. Micron 27:35-91. 